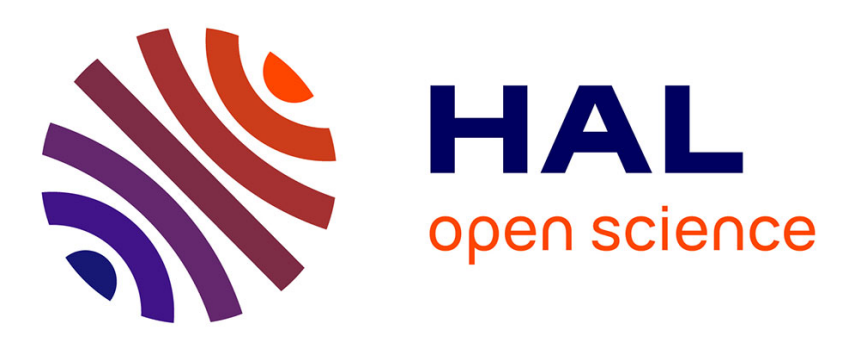

\title{
Tracking cells in the brain of small animals using synchrotron multi-spectral phase contrast imaging
}

Clément Tavakoli, Elisa Cuccione, Chloe Dumot, David Cormode, Marlène Wiart, Hélène Elleaume, Emmanuel Brun

\section{- To cite this version:}

Clément Tavakoli, Elisa Cuccione, Chloe Dumot, David Cormode, Marlène Wiart, et al.. Tracking cells in the brain of small animals using synchrotron multi-spectral phase contrast imaging. SPIE Medical Imaging,, Feb 2021, Online Only, France. pp.164, 10.1117/12.2580841 hal-03428586

\section{HAL Id: hal-03428586 \\ https://hal.science/hal-03428586}

Submitted on 15 Nov 2021

HAL is a multi-disciplinary open access archive for the deposit and dissemination of scientific research documents, whether they are published or not. The documents may come from teaching and research institutions in France or abroad, or from public or private research centers.
L'archive ouverte pluridisciplinaire HAL, est destinée au dépôt et à la diffusion de documents scientifiques de niveau recherche, publiés ou non, émanant des établissements d'enseignement et de recherche français ou étrangers, des laboratoires publics ou privés. 


\title{
Tracking cells in the brain of small animals using synchrotron multi- spectral phase contrast imaging
}

\author{
Clément Tavakoli ${ }^{\mathrm{a}, \mathrm{b}}$, Elisa Cuccione ${ }^{\mathrm{a}}$, Chloé Dumot $^{\mathrm{a}}$, David Cormode $^{\mathrm{c}}$, Marlène Wiart ${ }^{\mathrm{a}}$, Hélène \\ Elleaume $^{\mathrm{b}}$, Emmanuel Brun ${ }^{\mathrm{b}}$ \\ ${ }^{a}$ CarMeN Laboratory, Institut National de la Santé et de la Recherche Médicale U1060, INRA

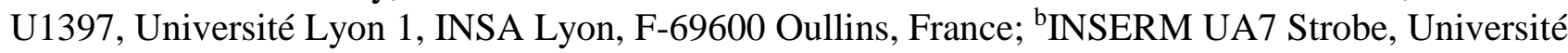 \\ Grenoble Alpes, ESRF, Grenoble, France; 'Department of Radiology, University of Pennsylvania, \\ Pennsylvania, United States
}

\begin{abstract}
Synchrotron X-ray multi-spectral imaging is a novel imaging modality that may allow tracking cells at high resolution in small animal models. The data volume generated by such technique can be of hundreds of Gigabytes for one animal. Automatic, robust and rapid pipeline is therefore of paramount importance for large-scale studies. The goal of this article is to present a full image analysis pipeline ranging from the CT reconstruction up to the segmentation of nanoparticleslabeled-cells. Experimentally, rats that had received an intracerebral transplantation of gold nanoparticles-labeled cells were imaged in vivo in phase contrast mode (propagation-based imaging technique) at two different energies strategically chosen around the k-edge of gold. We apply a dedicated phase retrieval technique on each projection (out of 2000 for complete $2 \pi$ rotation) before CT reconstruction. Then, a rigid registration is performed between the images below and above k-edge for accurate subtraction of the two data sets, leading to gold concentration maps. Due to the large number of specimens, the registration is based on the automatic segmentation of the cranial skull. Finally, an automatic segmentation of gold-labeled cells within the brain is performed based on high spots of gold concentrations. An example of an in-vivo data set for stroke cell therapy is presented.
\end{abstract}

Keywords: X-ray spectral imaging, registration, brain imaging

\section{INTRODUCTION}

Because it is fast and cost-effective, conventional attenuation-based X-ray Computed Tomography may be used as a noninvasive method for following up cellular therapies in small animal models [1]. Unfortunately, this technique faces some limitations in terms of resolution and contrast. Moreover, for separating the signal of contrast agents from similar endogenous signals, it often requires to image the animal before and after contrast agent injection, which may be a problem in terms of dose. Novel experimental CT techniques, namely Phase Contrast [2] and Spectral Imaging [3], that have been introduced in synchrotrons in the past years and recently translated to the clinics[4], [5], may help to overcome those limitations. While they were developed in parallel, the aim of our study is to combine the two approaches for an improved segmentation of labelled-cells with a focus on small animal follow-up with a model of focal cerebral injury mimicking stroke infarct.

Stroke is the leading cause of long-term disability, resulting in an increasing need for a reliable and reproducible brain regeneration technique. Several types of regenerative therapies are currently being evaluated, the introduction of stem cells through intracerebral route proving to be associated with the best recovery to date compared to other administration routes. It is thus essential to develop imaging modalities that allow the monitoring of the administered cells in order to follow their fate in a non-invasive manner. Labelling the injected cells using gold nanoparticles (AuNPs) allows to monitor the cells with CT-based imaging techniques, in particular the novel approaches described above.

The data volume generated by such technique can be of hundreds of Gigabytes for one animal. Automatic, robust and rapid pipeline is therefore of paramount importance for large-scale studies. In this work, we have developed a pipeline devoted to the rigid segmentation of labelled cells. After a brief introduction of the physical phenomena we introduce the image 
processing techniques used in this study for the signal reconstruction method, we then describe the main features of our registration and segmentation approach.

\section{SYNCHROTRON PHASE CONTRAST MULTI-SPECTRAL IMAGING}

\subsection{Physical principles}

Material Decomposition, the task to separate X-ray images into two or more materials, is usually performed using the element specific energy-dependence of X-ray attenuation [6], materialized by the linear attenuation coefficient $\mu$ of the element. This technique is currently being adopted in the clinics using either dual energy CT or Spectral Photon Counting CT scanners [7]. Using a wave approach of light, X-ray Phase Contrast Imaging (PCI) is able not only to reconstruct the linear attenuation coefficient $\mu$, but also the phase shift caused by the sample that can be quantified using the refractive index decrement $\delta$ for a given element. Indeed, each element can be described by its refraction index $n=1-\delta+i \beta$ where $\beta$ is the absorption index. Figure 1a shows in log scale the dependence in energy of the $\delta$ and $\beta$ indices for 3 elements/tissues. As one can see, like the linear attenuation coefficient, the absorption index shows a discontinuity at the $\mathrm{K}$-edge of the element $(80.7 \mathrm{keV}$ for gold). One can note that the $\delta / \beta$ ratio can be up to 2180 for water at $40 \mathrm{keV}$ resulting in an increased contrast with phase sensitive imaging [2]. In the recent years, PCI has demonstrated, at multiple occasions, to increase the contrast in the images when compared to conventional attenuation-based CT imaging in several domains such as lungs [8], breasts [9] or joints [10].

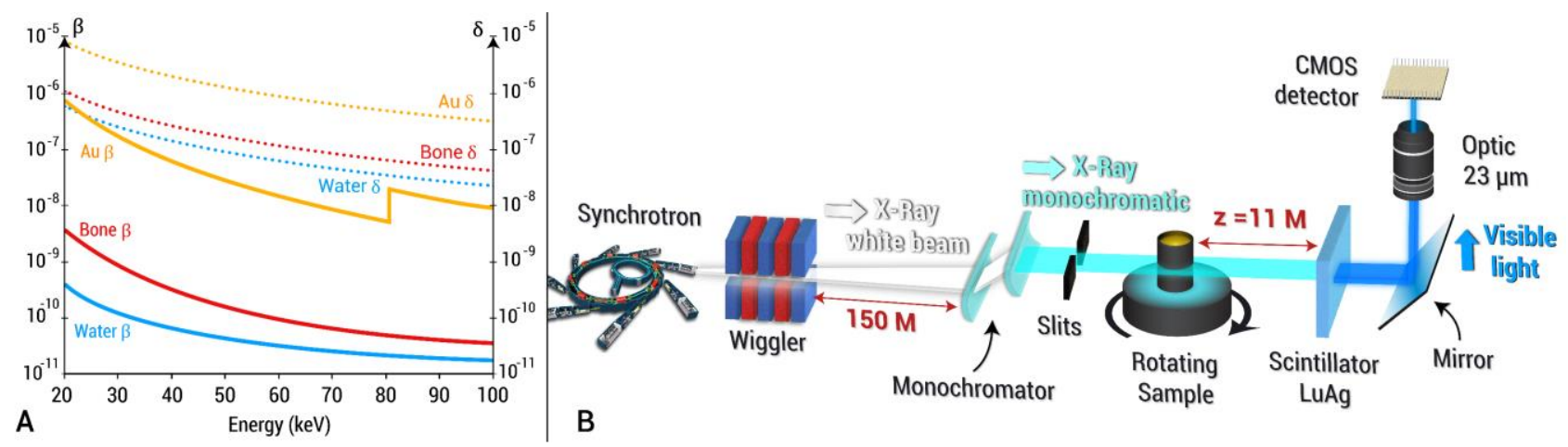

Figure 1. Combined Phase contrast and K-edge Imaging technique (A: Indices of refraction and absorption dependence in energy for water, bone and gold. B: Phase contrast imaging set-up).

Among all PCI experimental set-ups, here, we chose to use the Propagation Based Imaging [11] technique which has the great advantage, over other PCI setups, of being simple and fast for in vivo applications. The experimental set-up is shown in figure $1 \mathrm{~b}$. The only difference with a conventional X-ray imaging set-up is the additional distance $(\mathrm{z}=11 \mathrm{~m})$ between the sample and the detector. PBI explores the phase shifts caused by variations in the refractive index and density of materials by capturing alterations in the measured intensities which can be observed through edge enhancement. From the raw signal that contains both absorption and refraction one can use phase retrieval algorithms. To limit the computation time, we used a GPU version of the so-called Paganin algorithm [12]. This algorithm allows the retrieval of the linear attenuation coefficient $(\mu)$ assuming a unique $\delta / \beta$ ratio over the sample. Although this assumption is completely wrong in the case of radiography of biological samples mixing low and high absorbing tissue, it surprisingly works well in CT mode due to the fact that locally this unique $\delta / \beta$ ratio is true, as demonstrated in several studies [10], [13].

The second imaging technique, also referred to K-edge imaging, uses the discontinuity of the $\beta$ index around the K-edge of a specific element. In our study the cells were labelled with gold nanoparticles and CT images were acquired at two energies around the gold's K-edge. From these two acquisitions, after phase retrieval, we obtain 2 sets of data with linear coefficient $\mu_{\text {above }}$ and $\mu_{\text {below }}$ that we can estimate as:

$$
\left\{\begin{array}{l}
\mu_{\text {above }}=\mu_{M_{1}, E_{\text {above }}} \cdot f_{M_{1}}+\mu_{M_{2}, E_{\text {above }}} \cdot f_{M_{2}} \\
\mu_{\text {below }}=\mu_{M_{1}, E_{\text {below }}} \cdot f_{M_{1}}+\mu_{M_{2}, E_{\text {below }}} \cdot f_{M_{2}}
\end{array}\right.
$$


Where $f_{M_{1}}$ and $f_{M_{2}}$ are respectively the volume fraction of the material $M_{1}$ and $M_{2}$ of interest. $\mu_{M_{1}, E_{\text {above }}}$ is the linear coefficient for a given material at a given energy. So in our case, the concentration of gold can be retrieved by solving the linear system using the following formula:

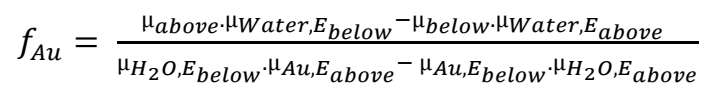

\subsection{Material}

We used the high intensity flux produced by the ESRF medical beamline (ID17) wiggler source which provides two quasicollimated monochromatic X-ray beams just below $(79.7 \mathrm{keV})$ and above $(81.7 \mathrm{keV})$ the K-edge of Gold $(80.7 \mathrm{keV})$. A total of eleven rats with a model of focal cerebral injury were studied. They were administered with 500k AuNP-labelled cells and imaged at the synchrotron ten days post-transplantation. Once anesthetized, the animals were mounted on a scanning sample stage located at approximately $11 \mathrm{~m}$ from the detector. Sets of 2000 projections were collected for each 360-degree rotation. An optical system consisting of a scientific CMOS technology-based camera coupled to a $\mathrm{Gd}_{2} \mathrm{O}_{2} \mathrm{~S}$ scintillator and an objective was used to record the X-ray images. The effective isotropic pixel size of the system was 21.3 $\mu \mathrm{m}$. Due to the small height of the beam $(4 \mathrm{~mm})$, multiple CT acquisitions were necessary to image the complete region of interests (maximum $17 \mathrm{~mm}$ in height). A total time of 12 minutes was necessary to collect the whole tomographic data set for one rat for the 2 data volumes. After phase retrieval a standard filtered back projection was used for the CT reconstructions, as described in [14].

\section{IMAGE ANALYSIS PIPELINE}

As the images were acquired in vivo sequentially, the rat movements had to be considered. Figure 2 presents the entire pipeline developed for the segmentation of AuNP-labelled cells. We chose to register the images based on the skull of the rats. This allowed us to select portions of the 3D data sets that are of interest, thereby reducing the size of the volumes to study and accelerate the speed of the registration algorithm. Finally, once the volumes have been registered and subtracted, the gold concentrations could be measured, corresponding to the AuNP-labelled cells injected into the brain. The details of each process of the pipeline is described in the following sub-sections.

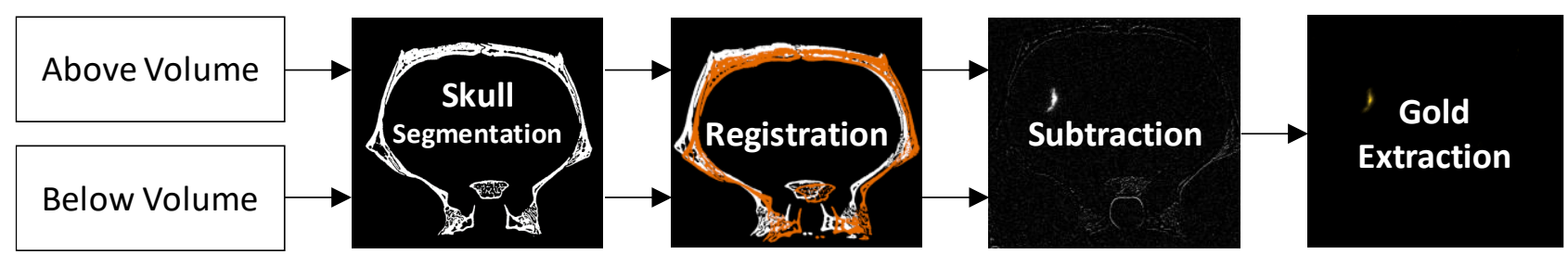

Figure 2. The 4 steps of the cells tracking pipeline resulting in a map of gold concentrations obtained from the two volumes acquired above and below the gold K-edge.

\subsection{Skull segmentation}

The phase contrast method allows us to easily identify the intensity values corresponding to the structures of interest in our images, in this context: the skull of the rat. In fact, as seen on Figure 3, an analysis of the volume histogram allows the distinction between three main intensity groups: the first one corresponding to the air, the second one to the soft tissues and the plastic supporting the rat, the last one to the bones and the gold in the injected cells.

An initial step consists in performing a threshold operation so that only the bone remains in the image. By performing a simple gradient analysis on the histogram, we can easily identify the peaks specific to the 3 parts described and thus calculate the optimal bone threshold value.

Once the bones have been segmented, we need to extract only the cranial skull and get rid of the other segmented structures such as jaws, labelled cells and teeth. To do so we labelled the different structures by a connected component analysis. For 
this purpose, we use the IPSDK library (Reactiv'IP, Grenoble France), which, as opposed to traditional libraries, has the great advantage of being adapted to large volumes, thus allowing us to gain a significant amount of time using three dimensional operations. We thereby end up with a list of labeled regions with characteristics associated to each of them, the size of which turns out to be an ideal discriminating factor for the identification of the cranial skull. Indeed, its size is at least ten times larger than the smaller structures (particularly the jaws). This method has proven to be robust for all the volumes studied at the different energies.
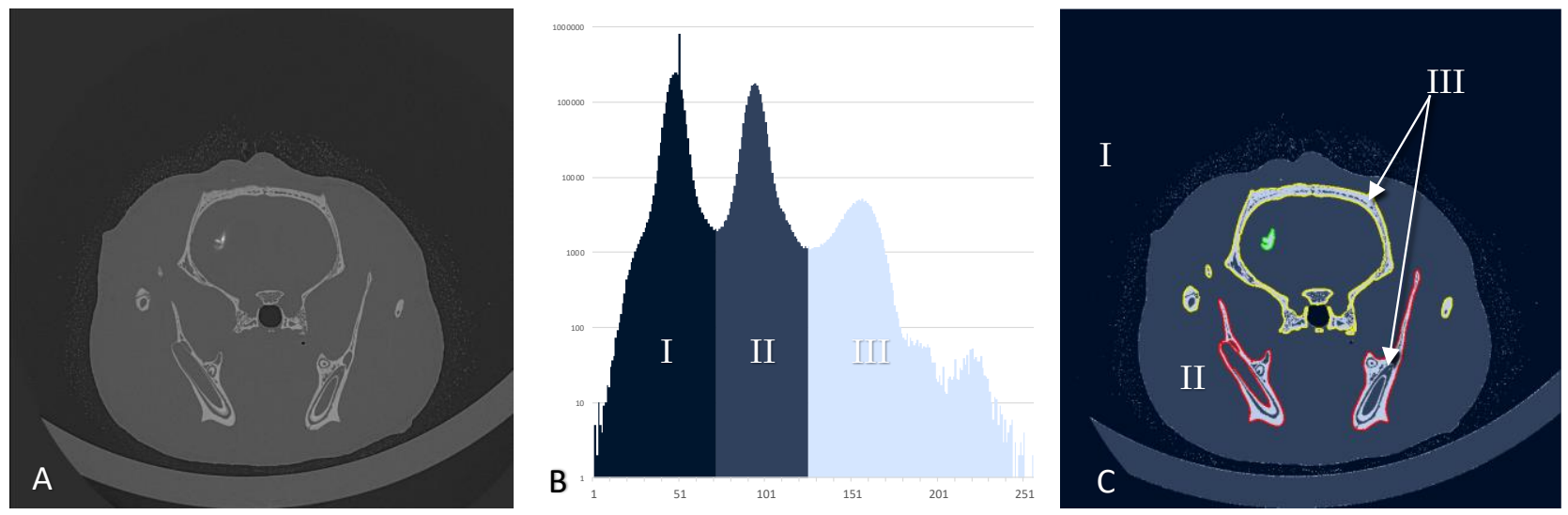

Figure 3. Intensity-based skull segmentation (A: Slice of the acquired volume, B: Histogram and its 3 intensity groups, C: Corresponding intensity regions (yellow: skull, red: jaws, green: gold)).

\subsection{Registration}

As explained previously, our intention consists in comparing two volumes $V_{\text {Below }}$ and $V_{\text {Above }}$ of the same sample acquired at two different energy levels $\left(\mathrm{E}_{\text {Below }}=79.7 \mathrm{keV}\right.$ and $\left.\mathrm{E}_{\text {Above }}=81.7 \mathrm{keV}\right)$. As can be seen on Figure 4, the variation in the position of the rat on the two volumes is too great to be neglected. The subfigure $4 \mathrm{c}$ shows indeed the subtraction of the 2 raw images ( $4 \mathrm{a}$ and $4 \mathrm{~b}$ ). It is therefore necessary to match the voxels representing the same point in space from one volume to the other and do the so-called registration of the two volumes.

Registration is a complex task with several parameters including the optimizer, the transformation, the interpolator and finally the similarity metric. If the transformation is trivially rigid in our case, finding the other parameters is not straightforward. We assume that, in our case, the volumes were acquired using the same modality and only differ by a basic geometric translation and rotation. We empirically chose to split this registration into two steps: a first transformation $T_{1}$ consisting of a simple and fast 3D translation closing most of the gap between the two volumes, followed by a second transformation $T_{2}$ applying a rotation and translation to the space given 3 Euler angles and a 3D translation.
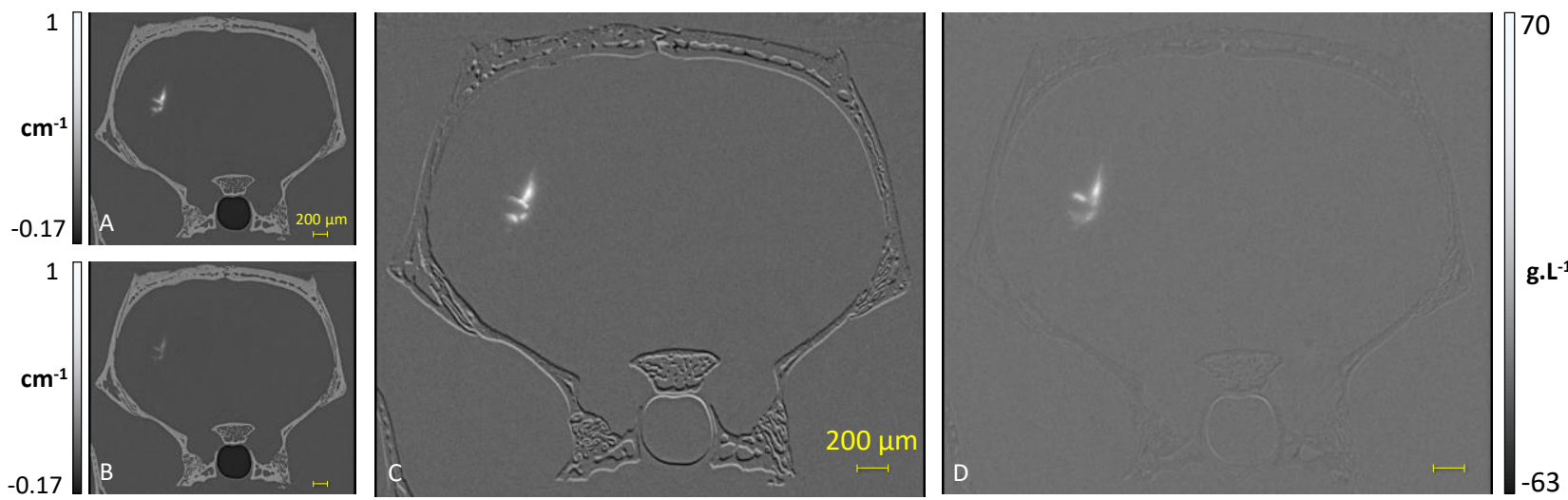

Figure 4. An example of the importance of registration for K-edge subtraction (A: Slice of $V_{\text {Above }}$, B: Slice of $V_{\text {Below }}$, C: Slice of the resulting K-edge subtraction without registration, D: Slice of the resulting K-edge subtraction with registration). The negative concentrations are caused by non-superimposed elements (hair in $\mathbf{C}$ and $\mathbf{D}$, bones, gold in $\mathbf{C}$ ). 
We chose to compare two typical intensity-based metrics such as the mean squared intensity difference (MSQ) and crosscorrelation (CC) in terms of rapidity to converge and final registration quality. While CC gave the best results for the optimization of $T_{1}$, the task for $T_{2}$ revealed to be more challenging. Therefore, in addition to these two metrics, we also evaluated the Mutual Information (MI) metric and the ANTs cross-correlation (ANTs CC). After some tests, we chose to use the gradient descent as an optimizer. Table 1 shows the results of this comparison on two animals.

Table 1. Comparison of 4 similarity metrics in terms of speed and quality. Mean Square Difference produces close to best results in the shortest period of time compared to other metrics.

\begin{tabular}{|c|c|c|c|c|c|}
\cline { 2 - 6 } \multicolumn{2}{c|}{} & CC & MSQ & ANTs CC (r=2) & MI \\
\hline \multirow{2}{*}{ Rat 1 } & Time (s) & 114 & $\mathbf{7 1}$ & 442 & 143 \\
\cline { 2 - 6 } & $\begin{array}{c}\text { Final } \\
\text { Error }\end{array}$ & $1.331 \%$ & $\mathbf{1 . 3 1 6 \%}$ & $1.401 \%$ & $1.328 \%$ \\
\hline \multirow{2}{*}{ Rat 2 } & Time (s) & 365 & $\mathbf{2 5 8}$ & 867 & 428 \\
\cline { 2 - 6 } & $\begin{array}{c}\text { Final } \\
\text { Error }\end{array}$ & $0.7214 \%$ & $0.7201 \%$ & $0.7401 \%$ & $\mathbf{0 . 7 1 9 6 \%}$ \\
\hline
\end{tabular}

The final error is calculated on the resulting subtraction of the superimposed volumes, using only the voxels contained in the cranial skull mask. We define it as follows:

$$
\text { Error }=\frac{N_{\text {error }}}{N_{\text {tot }}}
$$

Where $N_{\text {error }}$ is the number of voxels composing the mask with an absolute intensity above a threshold $\varepsilon=3 \sigma, \sigma$ being the intensity standard deviation among these voxels. $N_{\text {tot }}$ is the total number of voxels composing the mask.

As the table 1 shows, while the 4 methods produce comparable results in terms of quality, they differ when considering convergence speed. MSQ being less expensive to compute than both CC and MI, it is faster to converge. Given the fact there is no significant improvement using ANTs metric in this context, we opted for the MSQ metric defined as below:

$$
\operatorname{MSQ}(f, m)=\frac{\sum_{x y z}(f-m)^{2}}{n}
$$

Where $f$ and $m$ represent the vectors of the fixed and moving image intensities and $\mathrm{n}$ the total number of voxels.

\subsection{K-edge subtraction and gold extraction}

Once the volumes have been subtracted as defined in equation (2), we need to distinguish noise and artefacts from the AuNP-labelled cells. In order to do so, we begin with only keeping the parts corresponding to the brain by calculating the convex hull of the segmented cranial skull. We then subtract the dilated skull's mask leaving us with a limited number of structures, the biggest one corresponding to the brain. The next step consists in differentiating the gold NPs from the noise. We therefore apply a threshold defined as follows:

$$
\varepsilon_{\text {Gold }}=3 \sigma_{\text {Brain }}
$$

$\sigma_{\text {Brain }}$ being the standard deviation in the segmented brain. This operation is followed by a binary 3D opening with a 2 voxels radius sphere to remove the small structures that can be considered as noise. An example of result on rat 2 can be seen in Figure 5c. 

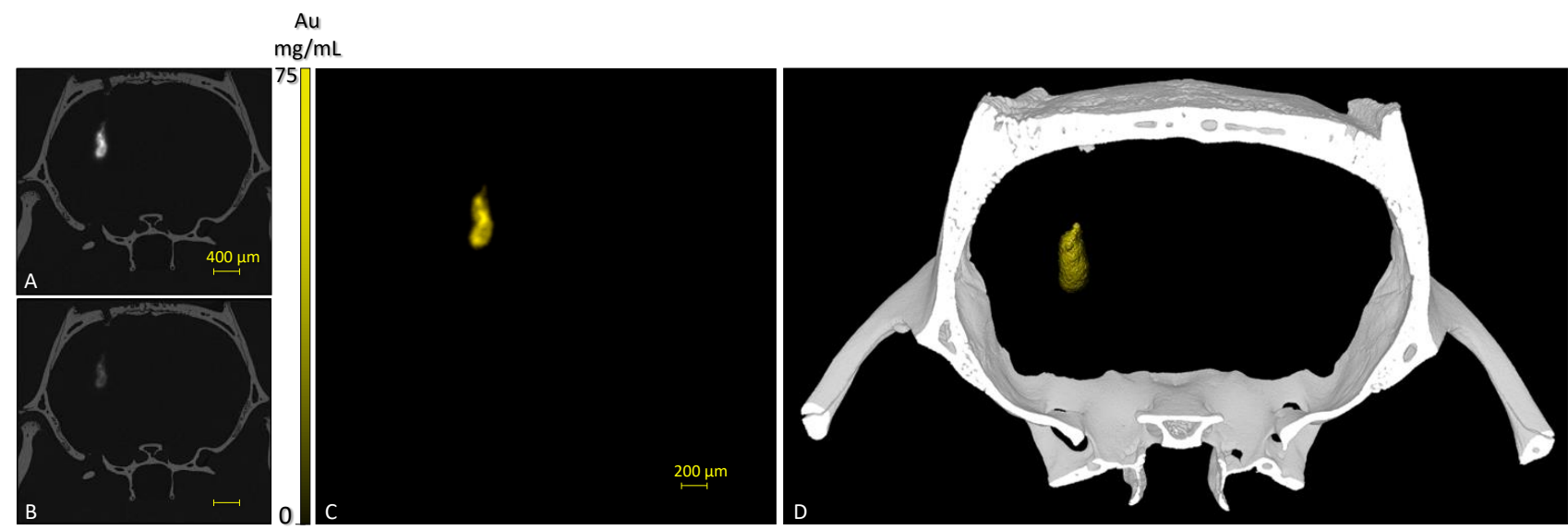

Figure 5. Results of gold extraction (A: Slice of $V_{A b o v e}$, B: Slice of $V_{B e l o w}$, C: Slice of the segmented gold in the resulting K-edge subtraction, D: 3D rendering of the segmented skull (white) and segmented gold (yellow))

\subsection{Time process}

Table 2 shows the computation time for the entire pipeline for the two rats presented above. The developed pipeline allows to have a total computation time in the same order as the acquisition time. The difference between the 2 rats is explained by the fact that rat 2 has moved less, only by a translation, and the algorithm tries nevertheless to improve the results by a rotation that is difficult to find and uses the maximum number of steps allowed in our implementation. Another explanation is the size of rat 2 cranial skull is bigger.

Table 2 : Time process (seconds) of each step of the developed pipeline, measured on 2 rats.

\begin{tabular}{|c|c|c|c|c|c|}
\cline { 2 - 6 } \multicolumn{1}{c|}{} & $\begin{array}{c}\text { Skull } \\
\text { Extraction }\end{array}$ & Registration & Subtraction & $\begin{array}{c}\text { Gold } \\
\text { Extraction }\end{array}$ & Total \\
\hline Rat 1 & $39.0 \mathrm{~s}$ & $208 \mathrm{~s}$ & $3.23 \mathrm{~s}$ & $80.1 \mathrm{~s}$ & $\mathbf{3 3 1 s}$ \\
\hline Rat 2 & $59.6 \mathrm{~s}$ & $419 \mathrm{~s}$ & $3.97 \mathrm{~s}$ & $92.9 \mathrm{~s}$ & $\mathbf{5 7 6 s}$ \\
\hline
\end{tabular}

\section{CONCLUSION}

In this work we have presented a whole image analysis pipeline for the automatic segmentation of spectral phase contrast imaging. The developed method uses the specificities of the synchrotron phase contrast signal to improve the contrast in our images. After some tests using different metrics, the registration method uses a gradient descent as optimizer, trilinear interpolation and cross correlation and mean squared difference as metrics. Once the spectral images are registered, we compute the maps of gold concentration in order to track the gold nanoparticles-labelled cells. We presented results on two rats but we tested our method on a whole dataset of in vivo rats with a focal cerebral injury mimicking stroke. The algorithm proved to be robust and fast on big datasets (several gigabytes per rat). The main limitation of this study is the fact that we only tested our pipeline on a cerebral application but we will use and test this pipeline on another medical disease (osteoarthritis) in the near future.

\section{REFERENCES}

[1] J. R. Ashton, J. L. West, and C. T. Badea, "In vivo small animal micro-CT using nanoparticle contrast agents," Front. Pharmacol., vol. 6, p. 256, Nov. 2015, doi: 10.3389/fphar.2015.00256.

[2] A. Bravin, P. Coan, and P. Suortti, "X-ray phase-contrast imaging: from pre-clinical applications towards 
clinics.," Phys. Med. Biol., vol. 58, no. 1, pp. R1-R35, Jan. 2013, doi: 10.1088/0031-9155/58/1/R1.

[3] W. Thomlinson, H. Elleaume, L. Porra, and P. Suortti, "K-edge subtraction synchrotron X-ray imaging in biomedical research," Phys. Medica, vol. 49, pp. 58-76, May 2018, doi: 10.1016/j.ejmp.2018.04.389.

[4] K. Willer et al., "X-ray dark-field imaging of the human lung - A feasibility study on a deceased body," PLoS One, vol. 13, no. 9, p. e0204565, Sep. 2018, doi: 10.1371/journal.pone.0204565.

[5] U. Fehrenbach et al., "Spectral CT and its specific values in the staging of patients with non-small cell lung cancer: technical possibilities and clinical impact," Clin. Radiol., vol. 74, no. 6, pp. 456-466, Jun. 2019, doi: 10.1016/j.crad.2019.02.010.

[6] R. E. Alvarez and A. Macovski, "Energy-selective reconstructions in X-ray computerised tomography," Phys. Med. Biol., vol. 21, no. 5, p. 002, Sep. 1976, doi: 10.1088/0031-9155/21/5/002.

[7] C. H. McCollough, S. Leng, L. Yu, and J. G. Fletcher, "Dual- and Multi-Energy CT: Principles, Technical Approaches, and Clinical Applications," Radiology, vol. 276, no. 3, pp. 637-653, Sep. 2015, doi: 10.1148/radiol.2015142631.

[8] M. J. Kitchen et al., "CT dose reduction factors in the thousands using X-ray phase contrast," Sci. Rep., vol. 7, no. 1, p. 15953, Dec. 2017, doi: 10.1038/s41598-017-16264-x.

[9] E. Castelli et al., "Mammography with Synchrotron Radiation: First Clinical Experience with Phase-Detection Technique," Radiology, vol. 259, no. 3, pp. 684-694, Jun. 2011, doi: 10.1148/radiol.11100745.

[10] H. Rougé-Labriet et al., "X-ray phase contrast osteo- articular imaging: a pilot study on cadaveric human hands," Sci. Rep., 2020.

[11] J. Baruchel et al., "X-ray optics and imaging with hard coherent synchrotron radiation," in Coherent X-Rays: Techniques and Applications, 1997, vol. 3154, pp. 51-62.

[12] D. Paganin, S. C. Mayo, T. E. Gureyev, P. R. Miller, and S. W. Wilkins, "Simultaneous phase and amplitude extraction from a single defocused image of a homogeneous object," J. Microsc., vol. 206, no. 1, pp. 33-40, Apr. 2002, doi: 10.1046/j.1365-2818.2002.01010.x.

[13] P. Tafforeau et al., "Applications of X-ray Synchrotron microtomography for non-destructive 3D studies of paleontological specimens," Appl. Phys. A, p. Accepted.

[14] A. Mirone, E. Brun, E. Gouillart, P. Tafforeau, and J. Kieffer, "The PyHST2 hybrid distributed code for high speed tomographic reconstruction with iterative reconstruction and a priori knowledge capabilities," Nucl. Instruments Methods Phys. Res. Sect. B Beam Interact. with Mater. Atoms, vol. 324, pp. 41-48, Jan. 2014, doi: 10.1016/j.nimb.2013.09.030. 